\title{
A Constructive Proof of the Heine-Borel Covering Theorem for Formal Reals
}

\author{
Jan Cederquist ${ }^{1}$ and Sara Negri ${ }^{2}$ \\ 1 Department of Computing Science \\ University of Göteborg \\ S-412 96 Göteborg, Sweden \\ e-mail ceder@cs.chalmers.se \\ 2 Dipartimento di Matematica Pura ed Applicata \\ Via Belzoni 7 - 35131 Padova, Italy \\ Department of Computing, Imperial College \\ 180 Queen's Gate, SW7 2BZ London, U.K. \\ e-mail negri@pdmat1.math.unipd.it
}

\begin{abstract}
The continuum is here presented as a formal space by means of a finitary inductive definition. In this setting a constructive proof of the Heine-Borel covering theorem is given.
\end{abstract}

\section{Introduction}

It is well known that the usual classical proofs of the Heine-Borel covering theorem are not acceptable from a constructive point of view (cf. [vS, F]). An intuitionistic alternative proof that relies on the fan theorem was given by Brouwer (cf. $[\mathrm{B}, \mathrm{H}]$ ). In view of the relevance of constructive mathematics for computer science, relying on the connection between constructive proofs and computations, it is natural to look for a completely constructive proof of the theorem in its most general form, namely for intervals with real-valued endpoints.

By using formal topology the continuum, as well as the closed intervals of the real line, can be defined by means of finitary inductive definitions. This approach allows a proof of the Heine-Borel theorem that, besides being constructive, can also be completely formalized and implemented on a computer. Formal topology can be expressed in terms of Martin-Löf's type theory; a complete formalization of formal topology in the ALF proof editor has been given in [JC]. A development of mathematical results in formal topology will then be a preliminary work for a complete formalization of these results. On the basis of the present work, the first author has implemented the proof of the Heine-Borel theorem for rational intervals.

Moreover, here as elsewhere (see for instance [C, C2, N, NV]), the use of a pointfree approach allows to replace non-constructive reasoning by constructive proofs.

We point out that a proof similar in spirit to our work was given by MartinLöf in [ML].

The paper is organized as follows: in Section 2 we provide all the preliminary definitions on formal topology to make the exposition self-contained; in Section 3 
the continuum is defined as a formal space by means of an inductive definition, equivalent to the one given in [NS] but more suitable for our purpose. As an aside, the definition provides an explicit description of its Stone compactification (cf. $[\mathrm{N}]$ ). Formal reals are also proved to be equivalent to real numbers à la Bishop. In the following section, the formal space of a closed interval with rational endpoints is defined. Formal intervals are then proved to coincide, when considered in the extensional way as sets of points, with the usual intervals of the real line. Finally, the Heine-Borel covering theorem is proved and the same is done, without any substantial difference, for intervals with real-valued endpoints.

\section{Preliminaries}

We recall here the basic theoretical background concerning formal topology. Further general information can be found in [S, SVV], whereas in [N, NV] the constructive character of this approach to topology is testified by applications to constructive pointfree proofs. In [NS], the theory of real numbers in the framework of formal topology is developed, but we also provide here all the definitions needed.

Formal topologies were introduced by Per Martin-Löf and Giovanni Sambin ([S, S1]) as a constructive approach to (pointfree) topology, in the tradition of Johnstone's version of the Grothendieck topologies [J] and Fourman and Grayson's Formal Spaces [FG], but using simpler technical devices and a constructive set theory based on Martin Löf's constructive type theory.

The definition of a formal topology is obtained by abstracting from the definition of a topological space $\langle X, \Omega(X)\rangle$, without mentioning the points. Since a point-set topology can always be presented using one of its bases, the abstract structure that we will consider is a commutative monoid $\left\langle S,{ }_{\mathcal{S}}, 1_{\mathcal{S}}\right\rangle$ where the set $S$ corresponds to the base of the point-set topology $\Omega(X), \cdot \mathcal{S}$ corresponds to the operation of intersection between basic subsets, and $1_{\mathcal{S}}$ corresponds to the whole collection $X$.

In a point-set topology any open set is obtained as a union of elements of the base, but union does not make sense if we refuse reference to points; hence we are naturally led to think that an open set may directly correspond to a subset of the set $S$. Let $c^{*}$ denote the element of the base which corresponds to the formal basic open $c$. Since there may be many different subsets of basic elements whose union is the same open set, we need an equivalence relation $\cong_{S}$ between two subsets $U$ and $V$ of $S$ such that $U \cong_{\mathcal{S}} V$ holds if and only if the opens $U^{*} \equiv \cup_{a \in U} a^{*}$ and $V^{*} \equiv \cup_{b \in V} b^{*}$ are equal. For this purpose we introduce an infinitary relation $\triangleleft_{\mathcal{S}}$, called cover, between a basic element $a$ of $S$ and a subset $U$ of $S$ whose intended meaning is that $a \triangleleft_{\mathcal{S}} U$ when $a^{*} \subseteq U^{*}$. The conditions we require of this relation are a straightforward rephrasing of the analogous set-theoretic situation.

Besides the notion of cover, we introduce a predicate $\operatorname{Pos}_{\mathcal{S}}(a)[a \in S]$ to express positively (that is without using negation) the fact that a basic open is not empty. 
Definition 1 Formal topology. A formal topology over a set $S$ is a structure

$$
\mathcal{S} \equiv\left\langle S, \cdot \mathcal{S}, 1_{\mathcal{S}}, \triangleleft \mathcal{S}, P_{o s_{\mathcal{S}}}\right\rangle
$$

where $\left\langle S, \cdot \mathcal{S}, 1_{\mathcal{S}}\right\rangle$ is a commutative monoid with unit, $\triangleleft_{\mathcal{S}}$ is a relation, called cover, between elements and subsets of $S$ such that, for any $a, b \in S$ and $U, V \subseteq$ $S$, the following conditions hold:

$$
\begin{aligned}
& \text { (reflexivity) } \frac{a \in U}{a \triangleleft_{\mathcal{S}} U} \\
& \text { (transitivity) } \frac{a \triangleleft_{\mathcal{S}} U \quad U \triangleleft_{\mathcal{S}} V}{a \triangleleft_{\mathcal{S}} V} \text { where } U \triangleleft_{\mathcal{S}} V \equiv(\forall u \in U) u \triangleleft_{\mathcal{S}} V \\
& \left(\cdot \text { - left) } \frac{a \triangleleft_{\mathcal{S}} U}{a \cdot_{\mathcal{S}} b \triangleleft_{\mathcal{S}} U}\right. \\
& \left(\cdot-\text { right) } \frac{a \triangleleft_{\mathcal{S} U} U \triangleleft_{\mathcal{S}} V}{a \triangleleft_{\mathcal{S}} U \cdot_{\mathcal{S}} V} \text { where } U \cdot_{\mathcal{S}} V \equiv\{u \cdot \mathcal{S} v \mid u \in U, v \in V\}\right.
\end{aligned}
$$

and $P_{0 s_{\mathcal{S}}}$ is a predicate on $S$, called positivity predicate, satisfying:

$$
\begin{aligned}
& \text { (monotonicity) } \frac{\operatorname{Pos}_{\mathcal{S}}(a) \quad a \triangleleft_{\mathcal{S}} U}{(\exists b \in U) \operatorname{Pos}_{\mathcal{S}}(b)} \\
& \text { (positivity) } \quad a \triangleleft_{\mathcal{S}}\{a\}^{+} \quad \text { where } \quad U^{+} \equiv\left\{b \in U \mid \operatorname{Pos}_{\mathcal{S}}(b)\right\} .
\end{aligned}
$$

All the conditions, except positivity, are a straightforward rephrasing of the preceding intuitive considerations. One reason to introduce positivity is that any non-positive basic open is covered by everything. Indeed, when $P o s_{\mathcal{S}}$ is a decidable predicate, positivity is equivalent to

$$
\frac{\neg \operatorname{Pos}_{\mathcal{S}}(a)}{a \triangleleft \mathcal{S} \emptyset}
$$

and this will be the case both for the topology of formal reals and for the topology of intervals with rational endpoint. Technically, positivity also allows proof by cases on $\operatorname{Pos}_{\mathcal{S}}(a)$ for deductions involving covers (for a detailed discussion cf. [SVV]).

We point out that we can dispense with the unit in the definition of formal topology without any substantial difference in the development of the theory. This choice will be pursued in the sequel.

In order to connect our pointfree approach to classical point-set topology, the notion of point has to be recovered. Since we reverse the usual conceptual order between points and opens, and take the opens as primitive, points will be defined as particular, well behaved, collections of opens. We recall here the definition of a (formal) point of a formal topology: 
Definition 2. Let $\mathcal{A} \equiv\langle S, \cdot, 1, \triangleleft, P o s\rangle$ be a formal topology. A subset $\alpha$ of $S$ is said to be a formal point if for all $a, b \in S, U \subseteq S$ the following conditions hold:

1. $1 \in \alpha$;

2. $\frac{a \in \alpha \quad b \in \alpha}{a \cdot b \in \alpha}$;

3. $\frac{a \in \alpha \quad a \triangleleft U}{(\exists b \in U)(b \in \alpha)}$;

4. $\frac{a \in \alpha}{\operatorname{Pos}(a)}$.

In order to maintain the usual intuition on points, in the sequel we will write $\alpha \Vdash a$ ( $\alpha$ forces $a$, or $\alpha$ is a point in $a$ ) in place of $a \in \alpha$. Moreover, when a singleton set occurs we will sometimes omit curly brackets, and write $a \triangleleft b$ for $a \triangleleft\{b\}$, and $U \cdot b$ for $U \cdot\{b\}$.

\section{The Continuum as a Formal Space}

Formal real numbers can be obtained as formal points of a suitable formal topology based on the rationals (cf. [NS]). We are adopting here a somewhat different approach to formal reals in comparison with the one given in [NS]. We have the same monoid operation and positivity predicate, and the covering relations are equivalent, but we dispense with the unit. By this approach we avoid adding top and bottom to the rational numbers. The following definition was proposed by Thierry Coquand in order to make inductive arguments easier. Technically, it is a finitary inductive definition, since each rule involved has only finitely many premises (cf. [A]). In fact, we do not need to close under the cover rules. Moreover, as we will see, the definition provides a simple presentation of the Stone compactification for the cover (cf. [N]).

Definition 3. The formal topology of formal reals is the structure

$$
\mathcal{R} \equiv\langle Q \times Q, \cdot, \triangleleft, P o s\rangle,
$$

where $Q$ is the set of rational numbers, $S \equiv Q \times Q$ is the Cartesian product. The monoid operation is defined by $(p, q) \cdot(r, s) \equiv(\max (p, r), \min (q, s))$; the cover $\triangleleft$ is defined by

$$
(p, q) \triangleleft U \equiv\left(\forall p^{\prime}, q^{\prime}\right)\left(p<p^{\prime}<q^{\prime}<q \rightarrow\left(p^{\prime}, q^{\prime}\right) \triangleleft_{f} U\right),
$$

where the relation $\triangleleft_{f}$ is inductively defined by

$$
\begin{aligned}
& \text { 1. } \frac{q \leq p}{(p, q) \triangleleft_{f} U} ; \\
& \text { 2. } \frac{(p, q) \in U}{(p, q) \triangleleft_{f} U} ;
\end{aligned}
$$




$$
\begin{aligned}
& \text { 3. } \frac{(p, s) \triangleleft_{f} U \quad(r, q) \triangleleft_{f} U \quad p \leq r<s \leq q}{(p, q) \triangleleft_{f} U} \text {; } \\
& \text { 4. } \frac{\left(p^{\prime}, q^{\prime}\right) \triangleleft_{f} U \quad p^{\prime} \leq p<q \leq q^{\prime}}{(p, q) \triangleleft_{f} U} .
\end{aligned}
$$

The positivity predicate is defined by

$$
\operatorname{Pos}(p, q) \equiv p<q .
$$

According to the intuitive set-theoretic reading of the definition of formal topology, the above definition amounts to the following: A basic open $(p, q)$ is covered by a family $U$ of basic opens if and only if all $\left(p^{\prime}, q^{\prime}\right)$ strictly included in $(p, q)$ are included in the union of a finite subfamily of $U$. The rest of this section will be devoted to proving that the above definition really defines a formal topology whose formal points correspond to constructive real numbers.

The usual definition of formal point of a formal topology, given in Section 2, specializes to the following one when considering the formal topology of formal reals $\mathcal{R}$.

Definition 4. A subset $\alpha$ of $S$ is a formal point of $\mathcal{R}$ if it satisfies

1. $(\exists p, q)(\alpha \Vdash(p, q))$;

2. $\frac{\alpha \Vdash(p, q) \quad \alpha \Vdash\left(p^{\prime}, q^{\prime}\right)}{\alpha \Vdash(p, q) \cdot\left(p^{\prime}, q^{\prime}\right)}$;

3. $\frac{\alpha \Vdash(p, q) \quad(p, q) \triangleleft U}{\left(\exists\left(p^{\prime}, q^{\prime}\right) \in U\right)\left(\alpha \Vdash\left(p^{\prime}, q^{\prime}\right)\right)}$;

4. $\frac{\alpha \Vdash(p, q)}{\operatorname{Pos}(p, q)}$.

We observe here that, since $\operatorname{Pos}(p, q)$ is decidable, the fourth rule is provable from the third. Let $\operatorname{Pt}(\mathcal{R})$ denote the formal points of $\mathcal{R}$, called formal reals.

We will now prove that both $\triangleleft$ and $\triangleleft_{f}$ are covers, the latter being the Stone compactification of the former.

Proposition 5. The relation $\triangleleft_{f}$ is a cover.

Proof. Before proving the cover rules for $\triangleleft_{f}$, we observe that the rule of - - right follows from the rule of localization $\frac{a \triangleleft U}{a \cdot b \triangleleft U \cdot b}$ since the base is a semilattice.

Reflexivity: By definition.

Transitivity: Suppose $(p, q) \triangleleft_{f} U$ and $U \triangleleft_{f} V$. Then it is straightforward by induction on the derivation of $(p, q) \triangleleft_{f} U$ that $(p, q) \triangleleft_{f} V$.

- - Left: By the fourth axiom since $p \leq \max (p, r)$ and $\min (q, s) \leq q$.

Localization: Suppose $(p, q) \triangleleft_{f} U$. Then we prove, by induction on the derivation of $(p, q) \triangleleft_{f} U$, that $(p, q) \cdot(r, s) \triangleleft_{f} U \cdot(r, s)$. We first observe that we can assume $r<s$, because if $s \leq r$ the claim follows trivially by the first rule. If $(p, q) \triangleleft_{f} U$ is derived by the first or the second axiom the claim is trivial. 
Suppose it is derived by the third axiom with the assumptions $p \leq t<v \leq q$, $(p, v) \triangleleft_{f} U$ and $(t, q) \triangleleft_{f} U$. If $s \leq t$ then $\min (v, s)=\min (q, s)$ and therefore $(p, v) \cdot(r, s)=(p, q) \cdot(r, s)$. From $(p, v) \triangleleft_{f} U$, by induction hypothesis, we have $(p, v) \cdot(r, s) \triangleleft_{f} U \cdot(r, s)$ thus $(p, q) \cdot(r, s) \triangleleft_{f} U \cdot(r, s)$. If $v \leq r$ then $\max (t, r)=\max (p, s)$ and the conclusion follows as above by applying inductive hypothesis to the premiss $(t, q) \triangleleft_{f} U$. Otherwise $\max (t, r)<\min (v, s)$ and we have, by induction hypothesis and the same rule, $(p, q) \cdot(r, s) \triangleleft_{f} U \cdot(r, s)$. If it comes from $\left(p^{\prime}, q^{\prime}\right) \triangleleft_{f} U$, with $p^{\prime} \leq p<q \leq q^{\prime}$, then by induction hypothesis we get $\left(p^{\prime}, q^{\prime}\right) \cdot(r, s) \triangleleft_{f} U \cdot(r, s)$ and since $\max \left(p^{\prime}, r\right) \leq \max (p, r)$ and $\min (q, s) \leq \min \left(q^{\prime}, s\right)$ we obtain by the same rule $(p, q) \cdot(r, s) \triangleleft_{f} U \cdot(r, s)$.

Moreover we have the following essential result:

Proposition6. The relation $\triangleleft_{f}$ is a Stone cover, i.e., a cover with the property that, for arbitrary $(p, q) \in S$ and $U \subseteq S,(p, q) \triangleleft_{f} U$ implies the existence of a finite subset $U_{0}$ of $U$ such that $(p, q) \triangleleft_{f} U_{0}$.

Proof. Suppose $(p, q) \triangleleft_{f} U$. Then we can find a finite subset $U_{0}$ of $U$ such that $(p, q) \triangleleft_{f} U_{0}$ by induction on the derivation of $(p, q) \triangleleft_{f} U$.

The following lemma is used to prove that $\triangleleft$ is a cover.

Lemma 7. Suppose $(p, q) \triangleleft_{f} U, U \triangleleft V$ and let $p<p^{\prime}<q^{\prime}<q$. Then $\left(p^{\prime}, q^{\prime}\right) \triangleleft_{f} V$.

Proof. By induction on the derivation of $(p, q) \triangleleft_{f} U$. If $p \geq q$ and $p<p^{\prime}<q^{\prime}<q$ we have $\left(p^{\prime}, q^{\prime}\right) \triangleleft_{f} U$ by axioms 1 and 4 . If $(p, q) \in U$ then by the assumption $U \triangleleft V$ we have $(p, q) \triangleleft V$ and therefore if $p<p^{\prime}<q^{\prime}<q,\left(p^{\prime}, q^{\prime}\right) \triangleleft_{f} V$. If $p \leq r<s \leq q,(p, s) \triangleleft_{f} U$ and $(r, q) \triangleleft_{f} U$ we distinguish two cases according to the position of $r, s$ with respect to $p^{\prime}, q^{\prime}$. In the first case $r<p^{\prime}$ or $q^{\prime}<s$, in the second $p^{\prime} \leq r<s \leq q^{\prime}$. Suppose $r<p^{\prime}$, then $r<p^{\prime}<q^{\prime}<q$ so from the assumptions $(r, q) \triangleleft_{f} U$ and $U \triangleleft V$ we get, by induction hypothesis, $\left(p^{\prime}, q^{\prime}\right) \triangleleft_{f} V$. If $q^{\prime}<s$ we conclude symmetrically. If $p^{\prime} \leq r<s \leq q^{\prime}$ we can find $r^{\prime}$, s' such that $r<r^{\prime}<s^{\prime}<s$. Therefore we have $p<p^{\prime}<s^{\prime}<s$ and $r<r^{\prime}<q^{\prime}<q$. By induction hypothesis the former, together with $(p, s) \triangleleft_{f} U$ and $U \triangleleft V$ gives $\left(p^{\prime}, s^{\prime}\right) \triangleleft_{f} V$ and the latter together with $(r, q) \triangleleft_{f} U$ and $U \triangleleft V$ gives $\left(r^{\prime}, q^{\prime}\right) \triangleleft_{f} V$. Since $p^{\prime} \leq r^{\prime}<s \leq q^{\prime}$ we get the conclusion $\left(p^{\prime}, q^{\prime}\right) \triangleleft_{f} V$. If $(p, q) \triangleleft_{f} U$ is derived by the fourth rule we just apply induction hypothesis to the premiss and the fourth rule again.

Proposition 8. The relation $\triangleleft$ is a cover.

Proof. Reflexivity: Let $(p, q) \in U$, then $(p, q) \triangleleft_{f} U$ and so if $p<p^{\prime}<q^{\prime}<q$ we have $\left(p^{\prime}, q^{\prime}\right) \triangleleft_{f} U$. Therefore $(p, q) \triangleleft U$.

Transitivity: Let $p<p^{\prime}<q^{\prime}<q$. Then there exist $p^{\prime \prime}$ and $q^{\prime \prime}$ such that $p<p^{\prime \prime}<p^{\prime}<q^{\prime}<q^{\prime \prime}<q$ and $\left(p^{\prime \prime}, q^{\prime \prime}\right) \triangleleft_{f} U$. By the lemma above we have $\left(p^{\prime}, q^{\prime}\right) \triangleleft_{f} V$ and therefore $(p, q) \triangleleft V$.

- - Left: Suppose $(p, q) \triangleleft U$, then $(p, q) \cdot(r, s) \triangleleft U$ follows directly from the definitions since $\max (p, r)<p^{\prime}<q^{\prime}<\min (q, s)$ implies $p<p^{\prime}<q^{\prime}<q$.

- Right: Straightforward from the validity of - - right for $\triangleleft_{f}$. 
Finally, it is straightforward to prove monotonicity and positivity for Pos, thus completing the proof that $\mathcal{R}$ is a formal topology.

We will now prove that the cover $\triangleleft_{f}$ is the Stone compactification of the cover $\triangleleft$. We point out that this result is not needed in the proof of the HeineBorel theorem.

Proposition 9. If $(p, q) \triangleleft U$ and $U$ is finite, then $(p, q) \triangleleft_{f} U$.

Before proving Proposition 9, observe we can assume that, for all $(r, s) \in U$, $\operatorname{Pos}((p, q) \cdot(r, s))$ holds. In fact, if this is not the case, from $(p, q) \triangleleft U$ we have $(p, q) \triangleleft((p, q) \cdot U)^{+}$, and from $(p, q) \triangleleft_{f}((p, q) \cdot U)^{+}$, by - left and transitivity, $(p, q) \triangleleft_{f} U$. The following lemmas will allow a proof of Proposition 9 by induction on the number of elements of $U$.

Lemma 10. For positive $(p, q),(p, q) \triangleleft_{f}(r, s)$ implies $r \leq p<q \leq s$.

Proof. By induction on the derivation of $(p, q) \triangleleft_{f}(r, s)$. If $(p, q) \triangleleft_{f}(r, s)$ is derived by the first or the second axiom, the claim holds trivially. If it is derived by the third axiom from $p \leq u<v \leq q,(p, v) \triangleleft_{f}(r, s),(u, q) \triangleleft_{f}(r, s)$, then by induction hypothesis we have $r \leq p<v \leq s, r \leq u<q \leq s$ and therefore $r \leq p<q \leq s$. If it follows from $p^{\prime} \leq p<q \leq q^{\prime}$ and $\left(p^{\prime}, q^{\prime}\right) \triangleleft_{f}(r, s)$ by the fourth axiom, then by induction hypothesis $r \leq p^{\prime}<q^{\prime} \leq s$ and therefore $r \leq p<q \leq s$.

Corollary 11. $(p, q) \triangleleft(r, s)$ implies $(p, q) \triangleleft_{f}(r, s)$.

Proof. Let $(p, q) \triangleleft(r, s)$. Then, for all $p^{\prime}, q^{\prime}$ such that $p<p^{\prime}<q^{\prime}<q$, we have $r \leq p^{\prime}<q^{\prime} \leq s$, and therefore $r \leq p<q \leq s$, hence $(p, q) \triangleleft_{f}(r, s)$.

Lemma 12. Suppose that $p<q$ and $(p, q) \triangleleft U$, where $U$ is finite and for all $(r, s) \in U, \operatorname{Pos}((p, q),(r, s))$ holds. Then there exists $\left(p_{1}, q_{1}\right) \in U$ such that $p_{1} \leq p<q_{1}$.

Proof. Let $\left(p_{1}, q_{1}\right)$ be an element of $U$ such that $p_{1}$ is the smallest (with respect to the usual order of the rational numbers) of all the first projections of elements of $U$. Then $p_{1} \leq p$. In fact, for all $\left(p^{\prime}, q^{\prime}\right) \in U, p_{1} \leq \max \left(p^{\prime}, p\right)<\min \left(q^{\prime}, q\right) \leq q$, that implies $U \cdot(p, q) \triangleleft_{f}\left(p_{1}, q\right)$. Since $(p, q) \triangleleft U \cdot(p, q)$, we have by transitivity $(p, q) \triangleleft\left(p_{1}, q\right)$, and therefore, by Corollary 11 and Lemma 10 , we get $p_{1} \leq p<q$. Then, by the assumption that for, all $(r, s) \in U, \operatorname{Pos}((p, q) \cdot(r, s))$ holds, we have $p_{1} \leq p<q_{1}$.

Lemma 13. Suppose that $(p, q) \triangleleft_{f} U$, and let $p<u<q$. Then there exists $(r, s) \in U$ such that $r<u<s$.

Proof. Straightforward by induction on the derivation of $(p, q) \triangleleft_{f} U$.

Corollary 14. Suppose that $(p, q) \triangleleft U$, and let $p<u<q$. Then there exists $(r, s) \in U$ such that $r<u<s$. 
Proof. If $p<u<q$, there exist $p^{\prime}, q^{\prime}$ such that $p<p^{\prime}<u<q^{\prime}<q$ and therefore $\left(p^{\prime}, q^{\prime}\right) \triangleleft_{f} U$. Then the conclusion follows by Lemma 13 .

Lemma 15. Suppose that $(p, q) \triangleleft U$, and let $(r, s) \in U$ with $\neg P o s((p, q) \cdot(r, s))$. Then $(p, q) \triangleleft U \backslash\{(r, s)\}$.

Proof. From $(p, q) \triangleleft U$ we have, by positivity and - - right, $(p, q) \triangleleft(U \cdot(p, q))^{+}$. Since $\neg \operatorname{Pos}((p, q) \cdot(r, s))$ holds, we have $(U \cdot(p, q))^{+} \subseteq(U \backslash\{(r, s)\}) \cdot(p, q)$ and therefore $(p, q) \triangleleft(U \backslash\{(r, s)\}) \cdot(p, q)$, thus a fortiori $(p, q) \triangleleft U \backslash\{(r, s)\}$.

Proof of Proposition 9. The proof is by induction on the number of elements of $U$. If $U=\{(r, s)\}$ the claim follows by Corollary 11. Suppose the result holds for $|U|=n$ and suppose that $(p, q) \triangleleft U_{n+1}$, where $\left|U_{n+1}\right|=n+1$. By Lemma 12 there exists $\left(p_{1}, q_{1}\right) \in U_{n+1}$ such that $p_{1} \leq p<q_{1}$. If $q \leq q_{1}$ then $p_{1} \leq p<q \leq q_{1}$ and therefore $(p, q) \triangleleft_{f}\left(p_{1}, q_{1}\right)$, so by reflexivity and transitivity $(p, q) \triangleleft_{f} U_{n+1}$. Otherwise $q_{1}<q$, hence by Corollary 14 there exists $\left(p_{2}, q_{2}\right) \in U_{n+1}$ such that $p_{2}<q_{1}<q_{2}$. So we can find $r, s$ such that $q_{1}<r<s<q_{2}$. Since $p \leq r$ and $(p, q) \triangleleft U_{n+1},(r, q) \triangleleft U_{n+1}$. From $q_{1}<r$, we have $\neg P o s\left((r, q) \cdot\left(p_{1}, q_{1}\right)\right)$ and therefore, by Lemma 15 , we have $(r, q) \triangleleft U_{n+1} \backslash\left\{\left(p_{1}, q_{1}\right)\right\}$, so that by induction hypothesis $(r, q) \triangleleft_{f} U_{n+1} \backslash\left\{\left(p_{1}, q_{1}\right)\right\}$. Then a fortiori $(r, q) \triangleleft_{f} U_{n+1}$. Since $(p, s) \triangleleft_{f}\left\{\left(p_{1}, q_{1}\right),\left(p_{2}, q_{2}\right)\right\}$, we also have $(p, s) \triangleleft_{f} U_{n+1}$ and therefore $(p, q) \triangleleft_{f} U_{n+1}$.

We conclude this section with observing that formal reals offer an alternative approach to constructive analysis; they have been used in the treatment of the Hahn-Banach theorem (cf. [CCN]) and of the Cantor and Baire theorems (cf. [N1], [NS]). Moreover, we can show that they are equivalent to real numbers à la Bishop. First we recall the following (cf. [Bi]):

Definition 16. A real number is a sequence of rational numbers $\left(x_{n}\right)_{n}$ such that

$$
\left|x_{m}-x_{n}\right| \leq m^{-1}+n^{-1} \quad\left(m, n \in \mathbb{N}^{+}\right) .
$$

Two real numbers, $\left(x_{n}\right)_{n}$ and $\left(y_{n}\right)_{n}$, are equal if

$$
\left|x_{n}-y_{n}\right| \leq 2 n^{-1} \quad\left(n \in \mathbb{N}^{+}\right) .
$$

We have:

Proposition 17. There exists a bijective correspondence between formal reals and real numbers à la Bishop.

Proof. Let $\alpha$ be a formal real. By the rules in Definition $4, \alpha$ contains arbitrarily small intervals, in particular $(p, q)$ with $q-p \leq 2 / 3$. Since $\frac{2 x+y}{3}<\frac{x+2 y}{3}$ again by the rules in Definition 4, $\alpha \Vdash(x, y)$ implies $\alpha \Vdash\left(x, \frac{x+2 x}{3}\right) \vee \alpha \Vdash\left(\frac{2 x+y}{3}, y\right)$. Now we can recursively generate a sequence of intervals $\left(\left(x_{n}, y_{n}\right)\right)_{n}$, by case-analysis:

$$
\begin{aligned}
\left(x_{1}, y_{1}\right) & \equiv(p, q) \\
\left(x_{i+1}, y_{i+1}\right) & \equiv\left\{\begin{array}{l}
\left(x_{i}, \frac{x_{i}+2 y_{i}}{3}\right) \text { if } \alpha \Vdash\left(x_{i}, \frac{x_{i}+2 y_{i}}{3}\right) \\
\left(\frac{2 x_{i}+y_{i}}{3}, y_{i}\right) \text { if } \alpha \Vdash\left(\frac{2 x_{i}+y_{i}}{3}, y_{i}\right) .
\end{array}\right.
\end{aligned}
$$


It can be verified that the sequences $\left(x_{n}\right)_{n}$ and $\left(y_{n}\right)_{n}$ are real numbers according to Definition 16.

Conversely, if $\left(x_{n}\right)_{n}$ is a real number à la Bishop, then the set defined by

$$
\alpha \equiv \bigcup_{n \in \mathbb{N}^{+}}\left\{(p, q): p<x_{n}-2 / n<x_{n}+2 / n<q\right\}
$$

is a formal real.

Moreover the correspondence thus established is bijective.

\section{The Formal Space $[a, b]$}

Given two rational numbers $a, b$ such that $a<b$, we will define a formal space whose formal points are the formal points of $\mathcal{R}$ between $a$ and $b$. We will follow the standard way to build, from an open $U$ of a space $X$, a space classically corresponding to the closed subspace $X \backslash U$. Indeed, we will define a cover relation $\triangleleft_{[a, b]}$ and the intended meaning of $(p, q) \triangleleft_{[a, b]} U$ is that the part of $(p, q)$ inside the closed interval $[a, b]$ is covered by $U$. By classical set-theoretic reasoning we have that $(p, q) \cap[a, b] \subseteq \cup U$ is the same as

$$
(p, q) \subseteq(\cup U) \cup\{(r, a) \mid r<a\} \cup\{(b, s) \mid b<s\} .
$$

An interval $(p, q)$ is then positive in the space $[a, b]$ iff the part of $(p, q)$ inside $[a, b]$ is positive. This justifies the following:

Definition 18. Let $\mathcal{R} \equiv\langle Q \times Q, \cdot, \triangleleft, P o s\rangle$ be the formal topology of formal reals and let $[a, b]$ be defined by

$$
[a, b] \equiv\left\langle Q \times Q, \cdot \triangleleft_{[a, b]}, \operatorname{Pos}_{[a, b]}\right\rangle
$$

where the relation $\triangleleft[a, b]$ is defined by

$$
(p, q) \triangleleft_{[a, b]} U \equiv(p, q) \triangleleft U \cup\{(r, a) \mid r<a\} \cup\{(b, s) \mid b<s\},
$$

and the predicate $\operatorname{Pos}_{[a, b]}$ is defined by

$$
\operatorname{Pos}_{[a, b]}(p, q) \equiv \operatorname{Pos}((p, q) \cdot(a, b)) .
$$

In the sequel we will use the notation $\mathcal{C}[a, b]$ for $\{(r, a) \mid r<a\} \cup\{(b, s) \mid b<s\}$ and we will understand $\mathcal{C}[a, b]$ as the complement of $[a, b]$.

By the following proposition and by the immediate verification that $\operatorname{Pos}_{[a, b]}$ is a positivity predicate, the above does indeed define a formal topology.

Proposition 19. The relation $\triangleleft_{[a, b]}$ is a cover.

The proposition follows from the following lemma:

Lemma 20. Let $\triangleleft$ be a cover on the base $S$ and let $V \subseteq S$. Then the relation $\triangleleft_{V}$ defined by

$$
a \triangleleft_{V} U \equiv a \triangleleft U \cup V
$$

is a cover. 
Proof. Reflexivity, transitivity, - left are straightforward, and - - right follows from the fact that in general $(U \cup V) \cdot(W \cup V) \triangleleft(U \cdot W) \cup V$.

As in Section 3, the general definition of formal point of a formal topology can be specialized to $[a, b]$ :

Definition 21. A subset $\alpha$ of $S$ is a formal point of $[a, b]$ if it satisfies

1. $(\exists p, q)(\alpha \Vdash(p, q))$;

2. $\frac{\alpha \Vdash(p, q) \quad \alpha \Vdash\left(p^{\prime}, q^{\prime}\right)}{\alpha \Vdash(p, q) \cdot\left(p^{\prime}, q^{\prime}\right)}$;

3. $\frac{\alpha \Vdash(p, q) \quad(p, q) \triangleleft[a, b] U}{\left(\exists\left(p^{\prime}, q^{\prime}\right) \in U\right)\left(\alpha \Vdash\left(p^{\prime}, q^{\prime}\right)\right)}$;

4. $\frac{\alpha \Vdash(p, q)}{\operatorname{Pos}_{[a, b]}(p, q)}$.

As was the case in Definition 4 the fourth rule is provable from the third, since $\operatorname{Pos}_{[a, b]}(p, q)$ is decidable. We will denote with $\operatorname{Pt}([a, b])$ the collection of formal points of $[a, b]$, called formal reals of the interval $[a, b]$.

We recall here the definition of order for $P t(\mathcal{R})$ (cf. [NS]):

$$
\begin{gathered}
\alpha<\beta \equiv(\exists(p, q),(r, s) \in S)(\alpha \Vdash(p, q) \& \beta \Vdash(r, s) \& q<r) ; \\
\alpha \leq \beta \equiv \neg(\beta<\alpha) .
\end{gathered}
$$

Let $\bar{a}$ denote the formal point $\{(p, q) \mid p<a<q\}$, corresponding to the rational $a$. Then we have $\alpha<\vec{a} \Leftrightarrow(\exists(p, q) \in S)(\alpha \Vdash(p, q) \& q<a)$.

The following proposition says that the formal space $[a, b]$ really corresponds to the closed interval $[a, b]$, i.e., the definition of the formal space $[a, b]$ is correct:

Proposition 22. $\alpha \in P t([a, b]) \Leftrightarrow \alpha \in P t(R) \& \bar{a} \leq \alpha \leq \bar{b}$.

Proof. $\Rightarrow$ : Let $\alpha \in P t([a, b])$. It is immediate that $\alpha \in P t(R)$ since $(p, q) \triangleleft U$ implies $(p, q) \triangleleft_{[a, b]} U$. To show that $\bar{a} \leq \alpha$, suppose $\alpha<\bar{a}$. Then by definition $(\exists(p, q) \in S)(\alpha \Vdash(p, q) \& q<a)$ and therefore $\neg P o s_{[a, b]}(p, q)$, against the assumption. Hence $\bar{a} \leq \alpha$. The inequality $\alpha \leq \vec{b}$ is proved symmetrically.

$\Leftarrow$ : Let $\alpha \in P t(R) \& \bar{a} \leq \alpha \leq \bar{b}$. Clauses 1 and 2 are obvious.

3. Let $\alpha \Vdash(p, q)$ and $(p, q) \triangleleft_{[a, b]} U$. Then there exists $(r, s) \in U \cup \mathcal{C}[a, b]$ such that $\alpha \Vdash(r, s)$. Since $\operatorname{Pos}_{[a, b]}(r, s)$ holds, it cannot be $(r, s)=(r, a)$ or $(r, s)=(b, s)$ and therefore $(r, s) \in U$.

\section{The Heine-Borel Covering Theorem for $[a, b]$}

Here we will prove the Heine-Borel covering theorem asserting that any open cover of a closed and bounded interval has a finite sub-cover. We will use the notation $[a, b] \triangleleft_{[a, b]} U$ for $(\forall p, q)\left((p, q) \triangleleft_{[a, b]} U\right)$, meaning that $U$ covers the whole space $[a, b]$. 
Theorem 23. The formal space $[a, b]$ is compact, i.e.

$$
[a, b] \triangleleft_{[a, b]} U \Rightarrow\left(\exists U_{0} \subseteq_{\omega} U\right)\left([a, b] \triangleleft_{[a, b]} U_{0}\right) .
$$

The proof uses the following lemma:

Lemma 24. $[a, b] \triangleleft_{[a, b]} U \Leftrightarrow(\exists r, s)\left(r<a<b<s \&(r, s) \triangleleft_{f} U \cup \mathcal{C}[a, b]\right)$.

Proof. $\Rightarrow$ : By the hypothesis $[a, b] \triangleleft[a, b] U$, in particular there exist $p, q$ such that $p<a<b<q$ and $(p, q) \triangleleft_{[a, b]} U$. Then by definition

$$
\left(\forall p^{\prime}, q^{\prime}\right)\left(p<p^{\prime}<q^{\prime}<q \rightarrow\left(p^{\prime}, q^{\prime}\right) \triangleleft_{f} U \cup \mathcal{C}[a, b]\right) .
$$

By choosing $r$ and $s$ such that $p<r<a<b<s<q$, we can thus conclude $(\exists r, s)\left(r<a<b<s \&(r, s) \triangleleft_{f} U \cup \mathcal{C}[a, b]\right)$.

$\Leftarrow$ : Observe that $(r, s) \triangleleft_{f} U \cup \mathcal{C}[a, b]$ implies $(r, s) \triangleleft U \cup \mathcal{C}[a, b]$, that is $(r, s) \triangleleft_{[a, b]} U$. If $r<a<b<s$, for all $(p, q),(p, q) \triangleleft\{(p, a),(r, s),(b, q)\}$ holds, and therefore $(p, q) \triangleleft_{[a, b]}\{(r, s)\}$. The claim follows by transitivity of $\triangleleft_{[a, b]}$.

Proof of Theorem 23. Suppose $[a, b] \triangleleft[a, b] U$. Then by Lemma 24 there exists $r$ and $s$ such that $r<a<b<s \&(r, s) \triangleleft_{f} U \cup \mathcal{C}[a, b]$ and by Proposition 6 there exists a finite subset $W_{0}$ of $U \cup \mathcal{C}[a, b]$ such that $(r, s) \triangleleft_{f} W_{0}$. Now, since $W_{0}$ is a finite subset of $U \cup \mathcal{C}[a, b]$, we can find a finite subset $U_{0}$ of $U$ such that $W_{0} \subseteq_{\omega} U_{0} \cup \mathcal{C}[a, b]$. We get $(r, s) \triangleleft_{f} U_{0} \cup \mathcal{C}[a, b]$. So, by Lemma 24 again, $[a, b] \triangleleft[a, b] U_{0}$.

\section{The Formal Space $[\alpha, \beta]$}

Generalizing the formal space $[a, b]$ that corresponds to an interval with rational endpoints, we will define the formal space $[\alpha, \beta]$, with $\alpha$ and $\beta$ formal reals with $\alpha<\beta$, that corresponds to an interval with real endpoints. The cover for the formal space $[\alpha, \beta]$ is defined starting from $\triangleleft$, similarly to the cover for $[a, b]$ :

Definition 25. Let $\triangleleft_{[\alpha, \beta]}$ be the relation defined by

$$
(p, q) \triangleleft_{[\alpha, \beta]} U \equiv(p, q) \triangleleft U \cup \mathcal{C}[\alpha, \beta],
$$

where $\mathcal{C}[\alpha, \beta] \equiv\{(r, a) \mid r<a<\alpha\} \cup\{(b, s) \mid \beta<b<s\}$.

Proposition 26. The relation $\triangleleft_{[\alpha, \beta]}$ is a cover.

The proof is immediate by Lemma 20 .

Definition 27. A subset $\gamma$ of $S$ is a formal point of $[\alpha, \beta]$ if it satisfies

1. $(\exists p, q)(\gamma \Vdash(p, q))$;

2. $\frac{\gamma \Vdash(p, q) \quad \gamma \Vdash\left(p^{\prime}, q^{\prime}\right)}{\gamma \Vdash(p, q) \cdot\left(p^{\prime}, q^{\prime}\right)}$; 
3. $\frac{\gamma \Vdash(p, q) \quad(p, q) \triangleleft[\alpha, \beta] U}{\left(\exists\left(p^{\prime}, q^{\prime}\right) \in U\right)\left(\gamma \Vdash-\left(p^{\prime}, q^{\prime}\right)\right)}$

4. $\frac{\gamma \Vdash(p, q)}{p<q \& \alpha<\bar{q} \& \bar{p}<\beta}$.

We remark that the property $p<q \& \alpha<\bar{q} \& \bar{p}<\beta$ of the basic neighbourhood $(p, q)$ expresses the fact that $(p, q)$ has positive intersection with the interval $[\alpha, \beta]$. Nevertheless, we do not call it a positivity predicate, since the property of positivity does not seem to be constructively valid for this predicate.

The collection of formal points of $[\alpha, \beta]$ will be denoted $P t([\alpha, \beta])$. As in the case of the formal space $[a, b]$ we have:

Proposition 28. $\gamma \in P t([\alpha, \beta]) \Leftrightarrow \gamma \in P t(R) \& \alpha \leq \gamma \leq \beta$.

Proof. $\Rightarrow$ : If $\gamma \in P t([\alpha, \beta])$ it is immediate to show $\gamma \in P t(R)$ since $(p, q) \triangleleft U$ implies $(p, q) \triangleleft_{[\alpha, \beta]} U$. Now suppose $\gamma<\alpha$. Then by definition

$$
\left(\exists\left(p_{\gamma}, q_{\gamma}\right),\left(p_{\alpha}, q_{\alpha}\right) \in S\right)\left(\gamma \Vdash\left(p_{\gamma}, q_{\gamma}\right) \& \alpha \Vdash\left(p_{\alpha}, q_{\alpha}\right) \& q_{\gamma}<p_{\alpha}\right) .
$$

From $\gamma \Vdash\left(p_{\gamma}, q_{\gamma}\right)$, by the fourth rule, we obtain that $\alpha<\bar{q}_{\gamma}$ which contradicts $\bar{q}_{\gamma}<\bar{p}_{\alpha}<\alpha$. Hence $\alpha \leq \gamma$. We obtain $\gamma \leq \beta$ symmetrically.

$\Leftarrow: 1$ and 2 are direct.

3. Let $\gamma \Vdash(p, q)$ and $(p, q) \triangleleft_{[\alpha, \beta]} U$. By definition we have $(p, q) \triangleleft U \cup \mathcal{C}[\alpha, \beta]$ and by the third rule for $P t(\mathcal{R})$ we get $\left(\exists\left(p^{\prime}, q^{\prime}\right) \in U \cup \mathcal{C}[\alpha, \beta]\right)\left(\gamma \Vdash\left(p^{\prime}, q^{\prime}\right)\right)$. If $\gamma \Vdash\left(p^{\prime}, q^{\prime}\right)$, by the fourth rule for $P t([\alpha, \beta])$ (which is proved below), $\alpha<\bar{q}^{\prime}$ and $p^{\prime}<\beta$ and therefore $\left(p^{\prime}, q^{\prime}\right) \in U$. Hence $\left(\exists\left(p^{\prime}, q^{\prime}\right) \in U\right)\left(\gamma \Vdash\left(p^{\prime}, q^{\prime}\right)\right)$.

4. Let $\gamma \|(p, q)$. Then by the fourth rule for $\operatorname{Pt}(\mathcal{R})$ we have $p<q$. If $\gamma \Vdash t(p, q)$ we also have $\gamma<\bar{q}$ and since $\alpha<\gamma$ we get $\alpha<\bar{q}$. The inequality $\bar{p}<\beta$ is proved symmetrically.

\section{The Heine-Borel Covering Theorem for $[\alpha, \beta]$}

Here we will prove the Heine-Borel covering theorem for closed intervals with real-valued endpoints. We introduce the notation:

$$
[\alpha, \beta] \triangleleft_{[\alpha, \beta]} U \equiv(\forall p, q)\left((p, q) \triangleleft_{[\alpha, \beta]} U\right) .
$$

Theorem 29. The formal space $[\alpha, \beta]$ is compact, i.e.

$$
[\alpha, \beta] \triangleleft_{[\alpha, \beta]} U \Rightarrow\left\{\exists U_{0} \subseteq_{\omega} U\right)\left([a, b] \triangleleft_{[\alpha, \beta]} U_{0}\right) .
$$

The proof uses the following lemma:

Lemma 30. $[\alpha, \beta] \triangleleft_{[\alpha, \beta]} U \Leftrightarrow(\exists r, s)\left(\bar{r}<\alpha<\beta<\bar{s} \&(r, s) \triangleleft_{f} U \cup \mathcal{C}[\alpha, \beta]\right)$. 
Proof. $\Rightarrow$ : Given $[\alpha, \beta] \triangleleft_{[\alpha, \beta]} U$, there exist $p, q$ such that $\bar{p}<\alpha<\beta<\bar{q}$ and $(p, q) \triangleleft_{[\alpha, \beta]} U$. By definition

$$
\left(\forall p^{\prime}, q^{\prime}\right)\left(p<p^{\prime}<q^{\prime}<q \rightarrow\left(p^{\prime}, q^{\prime}\right) \triangleleft_{f} \cup \cup \mathcal{C}[\alpha, \beta]\right)
$$

Now we can choose $r, s$ such that $\bar{p}<\bar{r}<\alpha<\beta<\bar{s}<\bar{q}$. Hence we obtain $(\exists r, s)\left(\bar{r}<\alpha<\beta<\bar{s} \&(r, s) \triangleleft_{f} U \cup \mathcal{C}[\alpha, \beta]\right)$.

$\nLeftarrow$ : Choose $(r, s)$ such that $\bar{r}<\alpha<\beta<\bar{s}$ and $(r, s) \nabla_{f} U \cup \mathcal{C}[\alpha, \beta]$. For any $a, b$ with $\bar{r}<\bar{a}<\alpha<\beta<\bar{b}<\bar{s}$ we get, for all $(p, q),(p, q) \triangleleft_{f}$ $\{(p, a),(r, s),(b, q)\}$. We have $(p, a) \triangleleft_{f} U \cup \mathcal{C}[\alpha, \beta]$ because if $p<a$ then $(p, a) \in$ $U \cup \mathcal{C}[\alpha, \beta]$ otherwise $(p, a) \triangleleft_{f} U \cup \mathcal{C}[\alpha, \beta]$ by axiom. By symmetry we have $(b, q) \triangleleft_{f} U \cup \mathcal{C}[\alpha, \beta]$, and therefore, by transitivity, $(p, q) \triangleleft_{f} U \cup \mathcal{C}[\alpha, \beta]$. This also means that $(p, q) \triangleleft_{[\alpha, \beta]} U$ and, since $(p, q)$ is arbitrary, $[\alpha, \beta] \triangleleft_{[\alpha, \beta]} U$.

Proof of Theorem 29. Suppose $[\alpha, \beta] \triangleleft_{[\alpha, \beta]} U$. Then, by Lemma 30, there exist $r$ and $s$ such that $\bar{r}<\alpha<\beta<\bar{s} \&(r, s) \triangleleft_{f} U \cup \mathcal{C}[\alpha, \beta]$ and by Proposition 5 there exists a finite subset $W_{0}$ of $U \cup \mathcal{C}[a, b]$ such that $(r, s) \triangleleft_{f} W_{0}$. Then we can find a finite subset $U_{0}$ of $U$ such that $W_{0} \subseteq_{\omega} U_{0} \cup \mathcal{C}[\alpha, \beta]$ and we get $(r, s) \triangleleft_{f} U_{0} \cup \mathcal{C}[\alpha, \beta]$. Using Lemma 30 again, $[\alpha, \beta] \triangleleft_{[\alpha, \beta]} U_{0}$.

\section{Acknowledgements}

We wish to thank Thierry Coquand and Jan Smith for helpful suggestions and remarks.

\section{References}

[A] P. Aczel. An Introduction to Inductive Definitions, in Handbook of Mathematical Logic, J. Barwise ed., North-Holland (1977) 739-782.

[B] L. E. J. Brouwer. Die intuitionistische Form des Heine-Borelschen Theorems, in L. E. J. Brouwer Collected Works, A. Heyting ed., North-Holland, Amsterdam (1975) vol. 1, 350-351, 1926C.

[Bi] E. Bishop. "Foundations of Constructive Analysis", Mc Graw Hill, 1967.

[JC] J. Cederquist. A machine assisted formalization of pointfree topology in type theory, Chalmers University of Technology and University of Göteborg, Sweden, Licentiate Thesis (1994).

[CCN] J. Cederquist, T. Coquand, S. Negri Helly-Hahn-Banach in formal topology, forthcoming.

[C] T. Coquand. An intuitionistic proof of Tychonoff's theorem, The Journal of Symbolic Logic vol. 57, no. 1 (1992) 28-32.

[C2] T. Coquand. Constructive Topology and Combinatorics, proceeding of the conference Constructivity in Computer Science, San Antonio, LNCS 613 (1992) 159-164.

[FG] M. P. Fourman, R.J. Grayson. Formal Spaces, in "The L. E. J. Brouwer Centenary Symposium", A. S. Troelstra and D. van Dalen (eds), North-Holland, Amsterdam (1982) 107-122. 
[F] M. Franchella. "L. E. J. Brouwer pensatore eterodosso. L'intuizionismo tra matematica e filosofia", Guerini Studio (1994).

[H] A. Heyting. "Intuitionism, an introduction", North-Holland (1971).

[J] P. T. Johnstone. "Stone Spaces", Cambridge University Press (1982).

[M] L. Magnusson, "The Implementation of ALF - a Proof Editor based on MartinLöf's Monomorphic Type Theory with Explicit Substitution", Chalmers University of Technology and University of Göteborg, $\mathrm{PhD}$ thesis (1995).

[ML] P. Martin-Löf. "Notes on Constructive Mathematics", Almqvist \& Wiksell, Stockholm (1970).

[ML1] P. Martin-Löf. "Intuitionistic type theory", notes by Giovanni Sambin of a series of lectures given in Padua, June 1980, Bibliopolis, Napoli (1984).

[N] S. Negri. Stone bases, alias the constructive content of Stone representation, "Logic and Algebra", A. Ursini and P. Aglianò eds., Dekker, New York (1996) 617-636.

[N1] S. Negri. "Dalla topologia formale all'analisi", Ph.D. thesis, University of Padua (1996).

[NS] S. Negri, D. Soravia. The continuum as a formal space, Rapporto Interno n.4, 17-7-95, Dipartimento di Matematica Pura e Applicata, Università di Padova.

[NV] S. Negri, S. Valentini. Tychonoff's theorem in the framework of formal topologies, The Journal of Symbolic Logic (in press).

[NPS] B. Nordström, K. Peterson, J. Smith, "Programming in Martin-Löf's Type Theory", Oxford University Press (1990).

[S] G. Sambin. Intuitionistic formal spaces - a first communication, in Mathematical logic and its applications, D. Skordev ed., Plenum (1987) 187-204.

[S1] G. Sambin. Intuitionistic formal spaces and their neighbourhoods, in "Logic Colloquium 88", R. Ferro et al. eds., North-Holland, Amsterdam (1989) 261-285.

[SVV] G. Sambin, S. Valentini, P. Virgili. Constructive Domain Theory as a branch of Intuitionistic Pointfree Topology, Theoretical Computer Science (in press).

[vS] W. P. van Stigt. "Brouwer's Intuitionism", Studies in the History and Philosophy of Mathematics, vol. 2, North-Holland (1990). 